\title{
Drug repurposing: cost effectiveness and impact on emerging and neglected diseases
}

\author{
Matthew Obaineh Ojezele ${ }^{1 *}$, Joseph Mordi $^{2}$ \& Emmanuel Adesola Adedapo ${ }^{3}$ \\ ${ }^{1}$ Department of Pharmacology \& Therapeutics, Delta State University, Abraka, Nigeria, \\ matlar2002@gmail.com, omojezele@,delsu.edu.ng, +2348033923332 \\ ${ }^{2}$ Department of Medical Biochemistry, Delta State University, Abraka, Nigeria, \\ drjoechuks@gmail.com, +2348038612368 \\ ${ }^{3}$ Department of Microbiology, University of Ibadan, Ibadan, Nigeria, emmasonline2015@gmail.com, \\ $+2348067806176$
}

\begin{abstract}
Historically, pressure on nature brought about by ever-increasing human pollution and technological advancement culminate in emergence and re-emergence of infectious and non-infectious diseases; necessitating medications and drug discovery and development. The emergence of resistant microorganisms and the emergence of new infections/disease conditions necessitate the production of entirely new drugs or modification of the existing ones to increase their efficacy. The development of novel medications is a very long and expensive process. There is a significant decrease observed in the number of new drugs approved for clinical use in recent years showing inconsistency in the face of scientific advances and research and development investment. Regardless of high investment and enormous contributions, very few molecules showed promising results. However, finding novel indications for existing drugs can be a useful method of reducing the developmental cycle of drugs. Repositioning (also called repurposing) has been described as the practice of developing new therapeutic uses for drugs, abandoned or drugs in development process, other than the initially intended or approved uses, except for the circumstances in which the novel use is comparable to the original indication with dissimilar pharmacological targets. This review aimed at looking into some of the available methods in drug repurposing.
\end{abstract}

Key words: Drug discovery; Drug repurposing; Machine learning; Neglected Tropical diseases; Orphan drugs

Received: 19/05/2020

Accepted: $10 / 07 / 2020$

DOI: https//dx.doi.org/10.4314/jcas.v16i1.1

(C) The Authors. This work is licensed under the Creative Commons Attribution 4.0 International Licence.

*Corresponding author: matlar2002@gmail.com 


\section{Resume}

Historiquement, la pression sur la nature provoquée par une pollution humaine et des progrès technologiques sans cesse croissants aboutit à l'émergence et à la réémergence de maladies infectieuses et non infectieuses; nécessitant des médicaments et la découverte et le développement de médicaments. L'émergence de micro-organismes résistants et l'émergence de nouvelles infections / maladies nécessitent la production de médicaments entièrement nouveaux ou la modification des médicaments existants pour augmenter leur efficacité. Le développement de nouveaux médicaments est un processus très long et coûteux. On observe une diminution significative du nombre de nouveaux médicaments approuvés pour usage clinique au cours des dernières années, montrant une incohérence face aux progrès scientifiques et aux investissements en recherche et développement. Malgré un investissement élevé et des contributions énormes, très peu de molécules ont montré des résultats prometteurs. Cependant, trouver de nouvelles indications pour des médicaments existants peut être une méthode utile pour réduire le cycle de développement des médicaments. Le repositionnement (également appelé repositionnement) a été décrit comme la pratique consistant à développer de nouvelles utilisations thérapeutiques pour des médicaments, abandonnés ou en cours de développement, autres que les utilisations initialement prévues ou approuvées, sauf dans les cas où la nouvelle utilisation est comparable à l'original indication avec des cibles pharmacologiques différentes. Cette revue visait à examiner certaines des méthodes disponibles en matière de réorientation des médicaments.

Mots clés: Découverte de médicaments; Réorientation des médicaments; Apprentissage automatique; Maladies tropicales négligées; Médicaments orphelins 


\section{Introduction}

The history of the use of drugs is as old as human existence. Historically, pressure on nature brought about by ever-increasing human pollution and technological advancement culminate in emergence and re-emergence of infectious and non-infectious diseases; necessitating medications and drug discovery and development. The emergence of resistant microorganisms and the emergence of new infections/disease conditions necessitate the production of entirely new drugs or modification of the existing ones to increase their efficacy.

The development of novel medications is a very long and expensive process; however, finding novel indications for existing drugs can be a useful method of reducing the developmental cycle of drugs (Zhao and So, 2017; Moreira de Oliveira and Lang, 2018). Drugs that have been repurposed can be in the market in a much shorter period and are inexpensive. The advent of omics (such as metagenomics, metabolomics and transcriptomics) and its rapid growth, other biomedical data in recent years, as well as computational drug repurposing offers a quick, inexpensive and organized means to determine promising repositioning prospects (Zhao and So, 2017). Medications that are not initially indicated for these diseases but with high predicted probabilities were used for repurposing as they are decent candidates for this purpose (Zhao and So, 2017).

The customary drug development procedure can be streamlined into two steps namely: Preclinical and Clinical. The preclinical step is based on the identification and molecule optimization, determination and validation of specific molecular target and the understanding of pharmacological as well as the toxicological implications via in vitro and in vivo models. This step aims to acquire a novel chemical entity having the capability to trigger a physiological action (Moreira de Oliveira and Lang, 2018). The clinical step is categorized into four subdivisions namely phase I, which is conducted in volunteers evaluating the rate of human tolerance of the novel drug as well as determining their safety dose (Brunton et al., 2012; Moreira de Oliveira and Lang, 2018). Phase II entails treatment efficacy and safety in the case of particular pathology as this is paramount for the continuity of the studies. Phase III evaluates the toxicity and safety of the novel chemical or drug, this is conducted in a cohort of larger volunteer patients close to the population for which the drug is intended, a comparison between the new and the current standard medication (Katzung et al., 2014; FDA, 2015). Phase IV is known as pharmacovigilance, after which the drug has been approved. This phase aims at a long-term assessment of certain parameters which are not observed in the previous phases as in the case of adverse reactions, hypersensitivity and drug interaction (Brunton, 2012; FDA, 2015; Moreira de Oliveira and Lang, 2018).

Regardless of high investment and enormous contributions, very few molecules showed promising results (Moreira de Oliveira and Lang, 2018). Research showed that only one out of the one million potential drugs has a profile entry in clinical studies; however, it is susceptible to a reasonable amount of failures. In the modern era, major development failure in the clinical stage occurs in phase II and III associated with safety and efficacy (Harrison, 2016). There is a significant decrease observed in the number of new drugs approved for clinical use in recent years showing inconsistency in the face of scientific advances and research and development investments (Moreira de Oliveira and Lang, 2018). This review aimed at looking into all the available methods in drug repositioning or repurposing. 


\section{DRUG REPURPOSING/ REPOSITIONING}

There is no consensus concerning the globally acceptable term for drug repurposing as a result, different terminologies such as repositioning, reformulating, and combination have been used by different researchers. Concerning these inconsistencies found in literature, Murteira et al., (2013) postulated a harmonized system of nomenclature for drug repositioning, reformulating, redirecting and combination. Repositioning (also called repurposing, re-tasking, re-profiling, or therapeutic switching) has been described as the practice of developing new therapeutic uses for drugs, abandoned or drugs in development process, other than the initially intended or approved uses, considering ICD-10, except for the circumstances in which the novel use is comparable to the original indication with dissimilar pharmacological targets (Murteira et al., 2013; Moreira de Oliveira and Lang, 2018). Lee and Kim (2016) opined that drug repurposing is a direct application of polypharmacology. Reformulation involves the development of novel inventions for medicinal drugs by taking the advantage of technological improvements towards the release of active substances, pharmaceutical forms and routes of administration. The combination involves mixing one or more drugs formally used as separate treatment which is a tactic for recurrent drug reuse in repositioning (Murteira et al., 2013).

The practice of drug repositioning is well known since the 1990s especially with the repositioning of thalidomide, a medicine known for its disaster when used as antiemetic in pregnant women as it showed teratogenic effects resulting in the birth of children with congenital deformities and its withdrawal from circulation in that same decade. However, Sampaio et al., (1991) demonstrated that thalidomide has selective inhibition of tumour necrosis factor (TNF- $\alpha$ ), cytosine found in leprous patients with elevated levels. Afterwards, this same drug was approved for the therapy of erythema nodosum in leprous patients by the Federal Drug Agency (FDA) (Wilkinson and Pritchard, 2015; Vianna et al., 2017). This same drug was once more repositioned for the therapy of refractory multiple myeloma (Singhal et al., 2000; Ashburn and Thor, 2004).

Another instance of drug repositioning was that of Sildenafil, a drug initially developed for antianginal activity. The drug, however, showed unsatisfactory cardiovascular effect but was also reported for unusually strong and persistent erections after usage. This drug was later approved as Sildenafil $\left(\right.$ Viagra $\left.^{\circledR}\right)$ the following year which later became a prominent medication for the treatment of erectile dysfunction (Ashburn and Thor, 2004). Furthermore, Sildenafil has been recently repositioned again as an orphan medication in the treatment of pulmonary hypertension (Wilkinson and Pritchard, 2015; Velayati et al., 2016; Moreira de Oliveira and Lang, 2018). Other drugs that have been repositioned include amantadine which was developed for influenza ab initio but redirected for Parkinson's disease. Furthermore, zidovudine was developed for cancer treatment. The drug was redirected towards HIV/AIDS (Lee and Kim, 2016) treatment.

\section{SIGNIFICANCE OF DRUG REPOSITIONING}

A propelling factor behind drug repositioning is the extension of application line or patent life of a drug thereby projecting investment return during the development process coupled with a higher rate of success and to further or continue its marketability (Parsaud-Sharma and Zhou, 2012). Additionally, some active molecules which might have been developed for other indications may be repositioned for the therapy of some neglected or rare disease conditions, which are usually 
difficult to tackle due to financial reasons (Sardana et al., 2011). The estimated cost of developing a new single drug is said to be US $\$ 800$ million with a duration of about ten to seventeen years while the cost of drug repositioning is just a fraction and the duration is far lesser than the formal duration as well as higher success rate (Parsaud-Sharma and Zhou, 2012). An instance is a statistical compilation reported which showed that the gross revenue attributed to Thalidomide, a repurposed drug indicated for multiple myeloma, surpassed US $\$ 271$ million in the year 2003 while Sildenafil or Viagra reached US\$1.88 billion in the same year (Dudley et al., 2011).

Furthermore, drug abandoning by pharmaceutical companies can be eradicated as there will be alternative indications even though the drug failed clinical trials for formal indications coupled with better financing for smaller companies; more so, new illnesses can be treated quickly (ParsaudSharma and Zhou, 2012). Conversely to the de novo drug development, drug repurposing or repositioning commences with known drug candidates and therefore, a substantial volume of data is available and accessible including the history of use, pharmacokinetics, bioavailability, toxicities including those that are common and uncommon, proven procedures and dosage, all of which are derived from phase I clinical trials of new drugs. However, phase I clinical trials may not be necessarily skipped and can be done to establish maximum dosage that can be tolerated. This dosage can be far higher than the standard dosage used in the initial drug indication if it is to reach oncological levels. If the repurposed drug is to be used in untried drug combination, there must be an establishment that there are no undesired toxicities (Pantziarka et al., 2014).

\section{DRUG REPOSITIONING PROFILES}

There are two major outlines to drug repurposing or repositioning which are 'On Target' and 'Off
Target'. The former deals with a known pharmacological mechanism of a candidate drug being applied to new therapeutic indications; in other words, the molecules that act on the same target in the treatment of different diseases (Koch et al., 2014). An instance of 'On Target' is the repositioning of finasteride which was initially meant for the therapy of benign hyperplasia because its pharmacokinetics leads to a reduction in dihydrotestosterone, an active metabolite of testosterone responsible for the growth of prostate growth by inhibiting an enzyme known as $5-\alpha$ reductase enzyme. Meanwhile, the inhibition of 5 - $\alpha$-reductase enzyme by this drug also reduces the bioavailability of dihydrotestosterone in the scalp leading to the shrinking of the hair follicle and so, finasteride is now subsequently used for the therapy of male pattern baldness medically known as alopecia; the drug has same target but different therapeutic effects.

The 'Off Target' entails unknown pharmacokinetics and therefore, the target and the mechanism of action are novel. This discovery is based on serendipity and several computational strategies employing several databases, algorithms and molecular coupling (Vora et al., 2016; Würth et al., 2016).

\section{METHODOLOGIES FOR DRUG REPOSITIONING}

These methodologies can be classified into three categories which are drug-oriented, target-oriented and disease or therapy-oriented. The application of these methodologies depends solely on the quantity and quantity of the pharmacological, toxicological as well as the biological information (Jin and Wong, 2014).

The drug-oriented methodology involves the characteristics of the molecule structure, off label use data, adverse effects and phenotypic screening being assessed. The screening is an approach to 
identifying molecules with biological effects in cell/animal assays to determine compounds that bring about some desired effect. This follows traditional pharmacology where minute data is available and until activities against phenotypes are detected, studies are carried out to determine the related biological targets (Moffat et al., 2017; Moreira de Oliveira and Lang, 2018).

Target-oriented methods entails in vivo and in vitro high content screening (HCS) and or high throughput screening (HTS) of drugs for the presence of a biomarker or protein of interest as well as in silico screening of compounds from drug libraries (Li et al., 2016; Bellomo et al., 2017). Target-based methods enhance the probability of drug discovery since most targets are related directly with disease mechanisms (Swinney, 2013). Conversely, if more disease information is available, drug repositioning can be channelled by diseases and or treatments, the information given by genomics, metabolomics, proteomics, or data about how drugs change phenotypes in diseases. The major methods applied are computational an example of which include network and pathway analysis comprising the construction of specific disease networks, bearing in mind the key targets, and detecting proteins linked to cell and metabolic pathways of interest. These methods help to have the understanding of the omics data, thereby allowing the unravelling of disease pathways as well as their pharmacological targets ( $\mathrm{Li}$ and Lu, 2013; Jin and Wong, 2014; Wu et al., 2017). Furthermore, some methods are based on bioinformatics and chemoinformatics incorporating a large data volume available for drugs and diseases such as off-label use, adverse effects, drug chemical structures, target drug networks, omics data, among others. These methods include a handful of known information that can improve the drug repositioning process
(Jin and Wong; Wu et al., 2017; Moreira de Oliveira and Lang, 2018).

\section{TOOLS IN DRUG REPOSITIONING}

Pharmacological databases are resources of importance for drug repositioning. They not only accrue drug property data but also include biological entities. Pharmacological data serve as the bedrock of many computational approaches (Xue et al., 2018). DrugBank is a resource that is both bioinformatics as well as cheminformatics which combines drug data with broad drug target information and many of the network-based approaches function based on this database achieving outstanding results (Gönen et al., 2012; Wu et al., 2014). Proteomics databases include protein-protein interaction (PPI) networks. They are essential resources for building heterogeneous networks such as drug-protein-disease networks. Munich Information Centre for Protein Sequences (MIPS) is one of the famous proteomic databases. Also, 2D topographical fingerprints and 3D conformations are available chemical-based database, as chemical information on drugs is usually applied for the prediction of novel drug structures. PubChem is also a database for chemical molecules structures as the database contains a large amount of $2 \mathrm{D}$ data that can be employed to measure drug similarity and to construct chemical networks (Bolton et al., 2008).

Medical literature databases such as PubMed are important tools for studies in drug repositioning as large amounts of medical and biological literatures containing novel biological entities relation. It comprises over 27 million biomedical literature citations from life science journals, MEDLINE and online books. Furthermore, citations may also include publisher websites and PubMed Central links to full-text content (Xue et al., 2018). 
COMPUTATIONAL APPROACHES TO DRUG REPOSITIONING

Systematic computational analyses are strong approaches in drug repositioning, permitting the prediction and identification of novel targets and therapeutic indications (Kim, 2015). Due to their ability to access large numbers of compounds and data relating to the target disease, informatics-based approaches can complement and ease drug repositioning efforts systematically (Kim, 2015). Computational repositioning success is dependent on several components such as the data quality and magnitude, appropriate analytical tools and knowledge integration derived from the analyses to ensure the effective formulation of new opportunity for drug repositioning (Kim, 2015). Several computational methods are employed to identify novel drugdisease connections including Transcriptomic Approaches and Genome-Wide Association Studies. Many of the available computational approaches stand on the principle of gene expression response of cell lines after treatment or joining many types of information about disease-drug relationships which can be categorized from diverse viewpoints. An example is the grouping of drug repositioning methods by some researchers according to the biological networks used while some classified it into two types viz data-driven and hypothesis-driven (Gönen et al., 2012; Xue et al., 2018).

Xue et al., (2018) categorized computational approaches into three categories viz networkbased approaches, text-mining approaches and semantic approaches (Xue et al., 2018). The network-based approaches are mostly used in drug repositioning as it possesses the ability to integrate multiple data sources. Some of these include network-based cluster approaches stirred by the fact that biological entities such as disease, drug and protein in the same module of biological networks share characteristic homology and network-based propagation approaches (Xue et al., 2018).

Network-based cluster approaches aim at detecting many modules, also called subnetworks, groups or cliques making use of cluster algorithms in relation to the topology structures of networks (Xue et al., 2018). These modules include diverse associations such as drug-disease, drug-drug or drug-target associations. The most common network-based cluster approaches include Density-based Spatial Clustering of Applications with Noise (DBSCAN), Clustering in QUEst (CLIQUE), statistical Information Grid (STING) and Ordering Points to Identify the Clustering Structure (OPTICS). However, they cannot identify overlapping clusters (Xue et al., 2018). Other network-based cluster approaches include $\mathrm{k}$-meand-based network cluster algorithm, Clustering with Overlapping Neighborhood Expansion (ClusterONE). The network-based propagation approaches have a workflow that prior information propagates to some sub-network nodes and all network nodes from the source node. These methods have been proven to perform well in identifying disease-genes, disease-targets and disease-drug associations (Emig et al., 2013). Local propagation approaches, in some cases, may not make correct predictions as it only takes into account partial information of the network (Mei et al., 2012). Conversely, global approaches perform better since it contains all information from the entire network.

The networks in network-based approaches can be divided into homogenous and heterogeneous. An example of the homogenous network is the construction of a disease pathway from proteinprotein interaction (PPI) network analysis. The homogenous approaches are used to identify drug targets involved with multiple pathways (Xue et al., 2018). Nonetheless, the PPI networks have a limitation in that they only include information 
about protein without additional information. The era of big data has brought about an accumulation of various medical data such as drugs, diseases and targets data which has made it possible to construct heterogeneous networks in the network-based approach (Xue et al., 2018). Heterogenous networks integrate multiple information sources including genomes, proteomes and metabolic pathways which typically contain two-layer (disease-drug) or three-layer (disease-drug-gene) associations (Wu et al., 2013).

Text-mining-based approaches have been developed to tackle the problem of extracting new and essential biological entity relationships from the literature. It was developed to mine new ideas from scientific literature and detect links between biological concepts or entities (Xue et al., 2018). Text mining (TM) has been defined as the discovery employing computer, new, previously unknown information by extracting information automatically from various written sources (Hearst, 1999). The biological text mining major channel includes four phases namely biological knowledge discovery (BKD), biological name entry recognition (BNER), information retrieval (IR) and biological information extraction (BIE) (Xue et al., 2018).

The Swanson ABC model is the foundation of text mining in the medical fields which states that if concept $A$ is linked with concept $B$, and concept $\mathrm{B}$ is linked with concept $\mathrm{C}$, therefore, concept A may have a novel link with concept $C$ (Xue et al., 2018). On this concept of text mining, many studies have applied text-mining techniques in drug repositioning. The research employed an approach to building disease-specific drug-protein connectivity maps infusing network-mining and text-mining where a disease-protein relationship is first extracted from molecular interaction networks via network-mining, after which drug terms indirectly linked with certain diseases such as Alzheimer's disease were searched in PubMed abstracts via text mining. In the final step, drugs and proteins could be connected through drugdisease or disease-protein associations ( $\mathrm{Li}$ et al., 2009). Consequently, diltiazem and quinidine are hypertension and arrhythmia drugs respectively but researchers have found that these drugs could be used to treat Alzheimer's disease (Xue et al., 2018).

Semantics-based approaches workflow involve three steps. First, biological entity relationships which are extracted from previous information in huge medical databases and is then used to build the semantic network. Next, semantics networks which is based on existing ontology are constructed using the prior information in the first step. Lastly, mining algorithms are aimed to predict new associations in the semantic network (Xue et al., 2018).

\section{NEGLECTED TROPICAL DISEASES}

Neglected tropical diseases (NTDs) are various groups of communicable diseases that are prevalent in subtropical and tropical conditions affecting over a billion people (Moreira de Oliveira and Lang, 2018). People in abject poverty, inadequate sanitation and living near infectious vectors, domestic animals and other livestock are worst affected (Peeling et al., 2017). These neglected diseases include tuberculosis, malaria, dengue fever which is of viral origin, Buruli ulcer and other diseases caused by eukaryotic parasites such as unicellular kinetoplastid protozoa or multicellular helminths- flukes, tapeworms and nematodes fall into this category (Wilkinson and Pritchard, 2015).

These diseases are referred to as neglected since those who need them are unable to pay the economic cost of treatment without any vision of recovering research and development costs. Also, safety profile and/or effectiveness is poorly 
established as well as possible drug resistance. Additionally, there is a very low commercial interest in developing medications for the therapy of these conditions as a result of the difficult and time-consuming nature, associated risks and poor return on investments (Berenstein et al., 2016).

Some of the specific challenges of repurposing approach when applied to Neglected Tropical Diseases (NTDs) field include throughput. Highthroughput biologically pertinent screening assays do not exist while the few hundreds of compounds available for screening match the low throughput of some biologically pertinent phenotypic screens such as those phenotypic screens for parasitic nematodes. Drug target is another specific challenge in the field of NTDs. This is because many causal agents of these diseases are eukaryotic. There is a true possibility that overlap exists between classes of drug target in the pathogens and the diseases targets in pharmacological pipelines and important pathogen targets are unveiled empirically. Struggles to select possible targets by computational methods continue (Li et al., 2011).

However, screening arrays of compounds active on mammalian targets has been proven to be productive. This is seen in the discovery of cysteine proteases inhibitors, HMGCoA reductase, as well as a range of kinases. Cysteine proteases have significant functional roles in eukaryotic pathogens; mammals would have serine proteases in place of cysteine proteases in some biological settings (Wilkinson and Pritchard, 2015). Mammalian capthesins are potent inhibitors against Trypanosoma cruzi causing Chagas disease, Plasmodium spp. causing malaria, and hookworm (Rosenthal et al., 2002; Vermeire et al., 2012).

Studies have shown that $\beta$-hydroxy- $\beta$-glutaminylCoA (HMGCoA) reductase inhibitors are active against schistosome parasites, however, more researches are ongoing (Vandewaa et al., 1989; Chen et al., 1990). More so, the employments of kinase inhibitors in the treatment of oncological origin have shown to be promising targets in parasitic protozoa having related kinases as in the case of kinetoplastid protozoa (Urbaniak et al., 2012).

Another successful drug repositioning on drug target networks (DTNs) include Miltefosine, initially developed as an antineoplastic compound but failed as it showed no satisfactory effect in phase II clinical trials was limited to topical therapy of cutaneous metastases is now repositioned for the therapy of visceral leishmaniasis (Barratt and Frail, 2012; Verhaar et al., 2014; Moreira de Oliveira and Lang, 2018). Also, amphotericin B, a drug formally developed for the treatment of systemic fungal infections but has been repositioned for the treatment of visceral leishmaniasis which was approved and supported by the phase IV clinical safety data accrued by the time of use as an antifungal (Barratt and Frail, 2012).

Zika virus has been a major menace in the class of NTDs facing Brazil amongst others such as dengue fever and chikungunya. Zika virus is associated with severe birth deformities and Guillain-Barré syndrome and there has not been an antiviral agent approved for the therapy of the viral infection (Moreira de Oliveira and Lang, 2018). Nonetheless, some drug repositioning researches have shown that screened FDA approved drugs for antiviral activity is a promising strategy for identifying drugs with novel activity for Zika virus infection (Tabata et al., 2016; Xu et al., 2016). Sofosbuvir (SOF) has been approved by the FDA for the therapy of hepatitis $\mathrm{C}$ which targets viral RNA polymerase, a common protein between hepatitis C and Zika virus. Also, sofosbuvir has been shown to exhibit protective 
activity for human neuronal progenitor cells and a reduction of viral load both in vivo and in vivo (Mesci et al., 2018).

Another promising drug repositioning evidence is the repositioning of a dopamine $\mathrm{D}_{2}$ receptor antagonist (prochlorperazine) approved for the therapy of headache, nausea and vomiting in humans showing strong in vivo and in vitro activity against dengue virus infection by targeting viral entry and binding (Simanjuntak et al., 2015).

\section{Rare and Orphan Disease Indications}

Rare diseases are those that are potentially fatal or chronic, usually hereditary, affecting a small number of people approximately 1 person in a population of 2000 (The European Commission on Public Health, 2016). In the United States, a rare disease is any disease or condition affecting less than 200, 000 people. In Japan, it is described as a disease that affects less than 50,000 patients while in Brazil a disease is considered rare when it affects up to 65 people in every 100,000 individuals (Barratt and Frail, 2012; Richter et al., 2015; Moreira de Oliveira and Lang, 2018). About 7000 rare and orphan diseases have been identified and they are classified as diseases that affect very few populations between $6 \%$ and $8 \%$ worldwide (Sadana et al., 2011; NIH, 2017).

The low population affected by these diseases presents poor prospects in the commercial industry in terms of returns while low prevalence and geographic dispersion of affected individuals of which many are children hampers efficient clinical trials (Wilkinson and Pritchard, 2015). Many of these diseases occur due to uncommon genetic disorders as well as mutations characterized by loss of functions in specific proteins that may not agree to small molecule therapies (Wilkinson and Pritchard, 2015; Hee et al., 2017). Hence, it is reasonable that repositioning can be a suitable approach than the de novo compound discovery.
Since there is prior knowledge about safety profiles, bioavailability, tolerance in humans which can reasonably reduce the risks linked with drug development and possibly facilitate the beginning of clinical trials, with reduced investment costs (Barratt and Frail, 2012; Wilkinson and Pritchard, 2015). Furthermore, combined computational approaches may be prolific in orphan diseases as a means of identifying repositioning prospects as well as target validation (Xu et al., 2011).

\section{Machine Learning Approach to Drug Repurposing in Psychiatry}

Psychiatric disorders have been identified as the leading causes of disabilities globally and there have been little improvements in the development of novel medications in the past decades. The development of new medications has also been limited by the challenge of animal models to completely mimic human psychiatric conditions. Furthermore, depression and anxiety disorders have been identified to show high clinical comorbidity (Lamers et al., 2011; Kessler et al., 2015), show substantial genetic correlation (Otowa et al., 2016), and share similar therapeutic pattern (Ballenger, 2000).

The machine learning approach is a universal and comprehensive structure that powers on machine learning methodologies as it is a field with exponential advances in the last decade (Zhao and So, 2017). This method offers boundless flexibility and chances for further development as any supervised learning method can be applied. Prediction algorithms that are newly developed can be integrated to enhance the detection of useful drugs. More so, in their method described, it applies to any chemical or drugs with expression profiles recorded, even without a known mechanism of action, as long as the transcriptomic profiling has been done, they can be analyzed for 
therapeutic or repurposing abilities (Zhao and So, 2017).

Zhao and So in 2017 presented a machine learning approach tailored towards predicting indication for drugs especially those indicated for schizophrenia and depression and anxiety disorders based on their expression profiles. They employed different machine learning approaches such as Deep Neural Networks (DNN), Support Vector Machine (SVM), Random Forest (RF), Gradient Boosted Machine with Trees (GBM) as well as logistic regression with Elastic Net Regularization (EN) to predict indications. After performing weighted and unweighted analysis, they found out that SVM showed overall best result for all un-weighted data analysis while DNN performed best for depression and anxiety disorders. Many of the drugs are antipsychotic such as trifluoperazine, perphenazine, fluphenazine and thioridazine, among others. Support Vector Machine and EN performed best for schizophrenia. Drugs listed for repositioning include Cyproheptadine and Chlorcyclizine which belong to the phenylpiperazine class antihistamine; Trichostatin A and vorinostat are top repositioning hits for depression/anxiety and schizophrenia (Zhao and So, 2017).

\section{CONCLUSION}

The idea of drug repositioning (repurposing, retasking, re-profiling, or therapeutic switching) is not new as it has been in vogue since the 1950s. It is a very potent approach to discovering new potential drugs for new and existing diseases as it consumes less time, high success rate, and lower financial input. It also increases the marketability of the drugs thereby increasing income for pharmaceutical companies. Neglected and rare or orphan diseases have a chance to be tackled through the application of drug repositioning having failed previously. Therefore, drug repositioning can be a tool for developing a new line of intervention for diseases that have been long forgotten and need appropriate and active treatments. However, the success of drug repositioning hinges on serendipity (polypharmacology or drug promiscuity). Various computational approaches are still under development to solving the health problems such as neurologic diseases as the signalling pathways and molecular mechanisms backing many disease states despite the rapid growth of advanced technology and the consequential molecular database making drug repositioning a difficult procedure.

\section{References}

Ashburn T.T., Thor K.B. Drug repositioning: identifying and developing new uses for existing drugs. Nature Review Drug Discovery. 2004; 3(8):673683.

Ballenger, J.C. Anxiety and Depression: Optimizing Treatments. Prim Care Companion Journal of Clinical Psychiatry 2, 71-79 (2000).

Barratt M.J., and Frail D.E. 2012. Drug Repositioning Bringing New Life To Shelved Assets And Existing Drugs. Hoboken, United States: John Wiley \& Sons.

Bellomo F., Medina D.L., De Leo E., Panarella A., Emma F. High-content drug screening for rare diseases. Journal of Inherited Metabolic Diseases. 2017; 40(4):601-607.

Berenstein A.J., Magariños M.P., Chernomoretz A., Agüero F. A Multilayer Network Approach for Guiding Drug Repositioning in Neglected Diseases. PLoS Neglected Tropical Diseases. 2016; 10(1):e0004300.

Bolton E., Yanli W., Paul. A.T., Stephen H. Bryant. PubChem: Integrated platform of small molecules and biological activities. Annual reports in computational chemistry. 2008;4: 217-41. 
Brunton LL, Chabner BA, Knollmann BC. 2012. Goodman And Gilman's The Pharmacological Basis of Therapeutics 12th edition. New York, United States: McGraw-Hill Medical.

Chen, G. Z.; Foster, L.; Bennett, J. L. Antischistosomal Action of Mevinolin: Evidence That 3-Hydroxy-Methylglutaryl-Coenzyme a Reductase Activity in Schistosoma mansoni Is Vital for Parasite Survival. Naunyn. Schmiedebergs Arch. Pharmacol. 1990, 342, 477-482.

Dudley J.T., Deshpande T., and Butte A.J. Exploiting drug-disease relationships for computational drug repositioning. Briefings in Bioinformatics. June 20, 2011; Vol. 12. No 4 303311.

Emig D., Ivliev A., Pustovalova O., Lancashire L., Bureeva S., Nikolsky Y., Bessarabova M. Drug target prediction and repositioning using an integrated network-based approach. PLoS ONE. 2013;8(4):e60618.

European Commission on Public Health. Rare diseases. Available at: https://ec.europa.eu/ health/rare_diseases/policy_en. [Accessed 10 October 2017].

FDA. 2015. The Drug Development Process. [Online] Available at: https://www.fda.gov/ ForPatients / Approvals/Drugs/default.htm. [Accessed 10 August 2017].

Gönen M. Predicting drug-target interactions from chemical and genomic kernels using Bayesian matrix factorization. Bioinformatics. 2012;28:230410.

Harrison RK. Phase II and phase III failures: 2013-2015. Nature Review Drug Discovery. 2016; 15(12):817-818.

Hearst M.A. Untangling text data mining. Proceedings of the 37 th annual meeting of the association for computational linguistics on computational linguistics: association for computational linguistics.1999;1:3-10.

Hee S.W., Willis A., Tudur Smith C., Day S., Miller F., Madan J., Posch M., Zohar S., Stallard N. Does the low prevalence affect the sample size of interventional clinical trials of rare diseases? An analysis of data from the aggregate analysis of clinicaltrials. Orphanet Journal of Rare Diseases. 2017; 12:44-63.

Jin G., and Wong S.T.C. Towards better drug repositioning: prioritizing and integrating existing methods into efficient pipelines. Drug Discovery Today, 2014; 19(5):637-644

Katzung BG, Trevor AJ, Masters SB. 2014. Basic and Clinical Pharmacology 12th Edition. New York: United States. McGraw-Hill Medical.

Kessler, R.C. et al. Anxious and non-anxious major depressive disorder in the World Health Organization World Mental Health Surveys . Epidemiol Psychiatr Sci 24, 210-26 (2015).

Kim Tae-Wan. Drug repositioning approaches for the discovery of new therapeutics for Alzheimer's disease. Neurotherapeutics (2015) 12:132-142

Koch U, Hamacher M, Nussbaumer P. Cheminformatics at the interface of medicinal chemistry and proteomics. Biochim Biophys Acta. 2014; 1844:156-161.

Lamers, F. Patricia van O., Hannie C.C., Johannes H.S., Philip S., Anton J.L.M., Willem A.N., Frans G.Z., Aartjan T.F.B., and Brenda W.J.H. Comorbidity patterns of anxiety and depressive disorders in a large cohort study: the Netherlands Study of Depression and Anxiety (NESDA). Journal of Clinical Psychiatry 72, 341-8 (2011). 
Lee Hyeong-Min and Kim Yuna. Review Article: Drug repurposing is a new opportunity for developing drugs against neuropsychiatric disorders. Schizophrenia Research and Treatment Vol. 2016, Article ID 6378137, 12 pages.

Li J., Lu Z. Pathway-based drug repositioning using causal inference. BMC Bioinformatics. 2013; 14(116):S3.

Li J., Zheng S., Chen B., Butte A.J., Swamidass S.J., Lu Z. A survey of current trends in computational drug repositioning. Briefings in Bioinformatics. 2016; 17(1):2-12.

Li J., Zhu X., Chen J.Y. Building disease-specific drug-protein connectivity maps from molecular interaction networks and PubMed abstracts. PLoS Computational Biology. 2009; 5: e1000450.

Li, Y. Y.; An, J.; Jones, S. J. A Computational Approach to Finding Novel Targets for Existing Drugs. PLoS Computational Biology. 2011, 7, e1002139.

Mei J.P., Kwoh C.K., Yang P., Li X.L., Zheng J. Drug -target interaction prediction by learning from local information and neighbors. Bioinformatics. 2013 Jan 15; 29(2):238-45. Doi: 10.1093/bioinformatics/bts670. Epub $2012 \mathrm{Nov}$ 17.

Mesci P., Macia A., Moore S.M., Shiryaev S.A., Pinto A., Huang C.T., Tejwani L., Fernandes I.R., Suarez N.A., Kolar M.J., Montefusco S., Rosenberg S.C, Herai R.H., Cugola F.R., Russo F.B., Sheets N., Saghatelian A., Shresta S., Momper J.D., Siqueira-Neto J.L., Corbett K.D., Beltrão-Braga P.C.B., Terskikh A.V., Muotri A.R. Blocking Zika virus vertical transmission. Sci Rep. 2018; 8(1):1218-1230.

Moffat J.G., Vicent F., Lee J.A., Eder J., Prunotto M. Opportunities and challenges in phenotypic drug discovery: an industry perspective. Nature Review Drug Discovery. 2017; 16(8):531-543.

Moreira de Oliveira E.A and Lang K.L. Drug repositioning: Concept, classification, methodology, and importance in rare/orphans and neglected diseases. Journal of Applied Pharmacentical Science Vol. 8(08), pp 157-165, August, 2018.

Murteira S, Ghezaiel Z, Karray S, Lamure M. Drug reformulations and repositioning in pharmaceutical industry and its impact on market access: reassessment of nomenclature. Journal of Market Access \& Health Policy. 2013; 1:21131.

NIH. 2017. National Center for Advaninc Translational Sciences. Genetic and rare diseases information center. Available at: https:// rarediseases.info.nih.gov/diseases. [Accessed 03 October 2017].

Otowa T. Hek K., Lee M., Byrne E.M., Mirza S.S., Nivard M.G., Bigdeli T., Aggen S.H., Adkins D., Wolen A. Fanous A., Keller M.C., Castelao E., Kutalik Z., Van der A., Homuth G., Nauck M., Teumer A., Milaneschi Y., Hottenga J.J., Direk N., Hofman A., Uitterlinden A., Mulder C.L., Henders A.K., Medland S.E., Gordon S., Heath A.C., Madden P.A., Pergadia M.L., van der Most P.J., Nolte I.M., van Oort F.V., Hartman C.A., Oldehinkel A.J., Presig M., Grabe H.J., Middeldorp C.M., Penninx B.W., Boosma D., Martin N.G., Montgomery G., Maher B.S., van der Oord E.J., Wray N.R., Tiemeier H., Hettema J.M. Metaanalysis of genome-wide association studies of anxiety disorders. Mol Psychiatry 21, 1485 (2016).

Pan, Y.; Cheng, T.; Wang, Y.; et al. Pathway Analysis for Drug Repositioning Based on Public Database Mining. J. Chem. Inf. Model. 2014, 54, 407-418. 
Pantziarka P., Gauthier B., Lydie M., Vidula S., Vikas P.S. The repurposing drugs in oncology (ReDO) project. ecancer medical science 2014, 8:442

Peeling R.W., Boeras D.I., Nkengasong J. Reimagining the future of diagnosis of Neglected Tropical Diseases. Comput Struct Biotechnol J. 2017; 15:271-274.

Persaud-Sharma Vishwani and Zhou Shu-Feng Drug repositioning: A faster path to drug discovery. Advances in Pharmacoepidemiology \& Drug Safety 2012, 1:6

Richter T., Nestler-Parr S., Babela R., Khan Z.M., Tesoro T., Molsen E., Hughes D.A. Rare Disease Terminology and Definitions-A Systematic Global Review: Report of the ISPOR Rare Disease Special Interest Group. Value Health. 2015; 18(6):906-914.

Rosenthal P.J., Sijwali P.S. Singh A., Shenai B.R. Cysteine: Proteases of Malaria Parasites: Targets for Chemotherapy. Curr. Pharm. Des. 2002, 8, 1659-1672.

Sampaio EP, Sarno EN, Galilly R, Cohn ZA, Kaplan G. Thalidomide selectively inhibits tumor necrosis factor alpha production by stimulated human monocytes. J Exp Med. 1991; 173(3):699703.

Sardana D., Zhu C., Zhang M., Gudivada R.C., Yang L., Jegga A.G. Drug repositioning for orphan diseases. Breifings in Bioinformatics. April 18, 2011; Vol. 12 No 4, 346-356.

Simanjuntak Y., Liang J.J., Lee Y.L., Lin Y.L. Repurposing of Prochlorperazine for Use Against Dengue Virus Infection. Journal of Infectious Diseases. 2015; 211(3):394-404.

Singhal S, Mehta J, Desikan R, Ayers D, Roberson P, Eddlemon P, Munshi N, Anaissie E, Wilson C,
Dhodapkar M, Zeddis J, Barlogie B. Antitumor Activity of Thalidomide in Refractory Multiple Myeloma. N Engl J Med. 2000; 341(21):15651571.

Swinney D.C. Phenotypic vs. Target-Based Drug Discovery for First-in-Class Medicines. Clin Pharmacol Ther. 2013; 93(4):299-301.

Tabata T., Petitt M., Puerta-Guardo H., Michlmayr D., Wang C., Fang-Hoover J., Harris E., Pereira L. Zika Virus Targets Different Primary Human Placental Cells Suggesting Two Routes for Vertical Transmission. Cell Host Microbe. 2016; 20(2):155166.

Urbaniak M.D., Mathieson T., Bantscheff M., Eberhard D., Grimaldi R., Miranda-Saavedra D., Wyatt P., Ferguson M.A, Frearson J., Drewes G. Chemical Proteomic Analysis Reveals the Drugability of the Kinome of Trypanosoma brucei. ACS Chem. Biol. 2012, 7, 1858-1865.

Vandewaa E.A., Mills G., Chen G.Z., Foster L.A., Bennett J.L. Physiological Role of HMG-CoA Reductase in Regulating Egg Production by Schistosoma mansoni. Am. J. Physiol.-Regul. Int. Am J Physiol. 1989, 257, R618-R625.

Velayati A, Valerio MG, Shen M, Tariq S, Lanier GM, Aronow WS. Update on pulmonary arterial hypertension pharmacotherapy. J Postgrad Med. 2016; 128(5):460-473.

Verhaar A.P., Wildenberg M.E., Peppelenbosch M.P., Hommes D.W., Van den Brink G,R. Repurposing Miltefosine for the Treatment of Immune- Mediated Disease? J Pharmacol Exp Ther. 2014; 350(2):189-195.

Vermeire, J. J.; Lantz, L. D.; Caffrey, C. R. Cure of Hookworm Infection with a Cysteine Protease Inhibitor. PLoS Negl. Trop. Dis. 2012, 6, e1680. 
Vianna FSL, Kowalski TW, Fraga LR, Sanseverino MT, Schuler- Faccini L. The impact of thalidomide use in birth defects in Brazil. Eur J Med Genet. 2017; 60(1):12-15.

Vora P.K., Somani R.R., Jain M.H. Drug Repositioning: An Approach for Drug Discovery. Mini-Reviews in Organ Würth ic Chemistry. 2016; 13(5):363-376.

Wilkinson Graeme F. and Pritchard Kevin. In vitro screening for drug repositioning. Journal of Biomolecular Screening 2015, Vol. 20(2) 167-179

Wu H, Gao L, Dong J, Yang X. Detecting overlapping protein complexes by rough-fuzzy clustering in protein-protein interaction networks. PLoS ONE. 2014;9(3):e91856.

Wu H, Huang J., Zhong Y., Huang Q. Drug Sig: A resource for computational drug repositioning utilizing gene expression signatures.. 2017 PLoS One; 12(5):e0177743.

Wu Z, Wang Y, Chen L. Network-based drug repositioning. Mol Biosyst. 2013; 9: 1268-81.
Würth, R, Thellung S, Bajettol A, Mazzanti M, Florio T, Barbieri F. Drug-repositioning opportunities for cancer therapy: novel molecular targets for known compounds. Drug Discovery Today. 2016; 21(1):190-199.

Xu, K.; Cote, T. R. Database Identifies FDAApproved Drugs with Potential to Be Repurposed for Treatment of Orphan Diseases. Briefings in Bioinformatics. 2011, 12, 341-345.

Xue H., Jie L., Haozhe X., and Yadong W. Review of drug repositioning approaches and resources. International Journal of Biological Sciences, 2018; 14(10): 1232-1244

Zhao Kai and So Hon-Cheong. A machine learning approach to drug repositioning based on drug expression profiles: Applications to schizophrenia and depression/anxiety disorders. KIZ-CUHK Joint Laboratory of Bioresources and Molecular Research of Common Diseases (2017). 